\title{
An Homogenization-Based Nonlocal Damage Model for Brittle Materials and Applications
}

\author{
Zhu Qizhi, Djimedo Kondo, and Shao Jian-fu* \\ Laboratory of Mechanics of Lille, URA CRNRS 8107, University of Lille, \\ Cité Scientifique, 59655 Villeneuve d'Ascq, France \\ jian-fu.shao@polytech-lille.fr
}

\begin{abstract}
A micromechanics-based constitutive damage model with clear physical grounds and few parameters is presented, in which the Ponte-Castaneda and Willis estimate (PCW estimate) is adopted and applied to solid matrix weaken by penny-shaped microcracks. The basic idea is to integrate the homogenization procedure into the standard thermodynamics framework. An energy release rate-based damage criterion is proposed in which the interaction effects between different cracks families are involved. The proposed constitutive model is then extended to an orientation-based nonlocal version. Finally, this model has been implemented into a finite element code and the numerical results obtained for $2 \mathrm{D}$ problems show its predictive capacity and mesh independent characteristics.
\end{abstract}

Keywords: Homogenization, damage, crack interaction, nonlocal.

\section{Introduction}

Nonlinear behavioral characteristics in brittle materials such as concrete and some rocks are commonly considered as the consequence of the nucleation and growth of microcracks [1] 2]. The modelling of the behaviors due to microcracking processes in brittle materials is a topic of great concern in many fields of civil engineering. Various material models have been developed, among which continuum damage mechanics based on the thermodynamics irreversible processes is widely used for investigation of materiral deteriorating phenomena by using one or several internal state-dependent variables [3] 4, 5. In spite of the efficiency of model implementations and the advantages in application to engineering analysis, some of the concepts and parameters involved in these models are not clearly related to physical mechanisms [6].

For two decades, several micromechanical models have been developed for concrete and rocks [7, 8, 9, which provide a promising tool for more physically based descriptions of damage induced anisotropy and of material degradation. However, these models are often limited to dilute distribution of microcracks without consideration of interactions between microcracks. Further, most of

\footnotetext{
^ Corresponding author.
} 
these models are not based on a rigorous upscaling method to define macroscopic properties of homogenized material. Moreover, these models without characteristic length leads to serious mesh dependency due to strain localization.

In this paper, materials weaken by microcracks are considered as a matrixinclusions (cracks) system [10]. The physically-based homogenization scheme developed by Ponte-Castaneda and Willis is adopted, in which the inclusions' geometry and the spatial distribution can be simultaneously taken into account by means of different functions [11]. The PCW estimate is then applied to pennyshaped microcracks and integrated into the standard thermodynamics framework. In addition, a strain energy release rate based damage criterion is used to determine the damage evolution in which interactions between microcracks are involved. In order to overcome the consequences due to the strain localization, a nonlocal formulation with material length is proposed. Finally, this model is implemented into a finite element code. The numerical results obtained from $2 \mathrm{D}$ problems show its predictive capacity and mesh independent characteristics.

\section{Model Formulations}

\subsection{Basic Principles of Upscaling Methods}

Consider the representative elementary volume (r.e.v.), occupying a domain $\Omega$ and having a boundary surface $\partial \Omega$, as a matrix-inclusion system, solid matrix with the elasticity tensor $\mathbb{C}^{s}$ and inclusions with $\mathbb{C}^{c, r}, r=1, \ldots, N$. The local behavior is assumed to be linear elastic: $\boldsymbol{\sigma}(\underline{z})=\mathbb{C}^{c}(\underline{z}): \varepsilon(\underline{z})(\forall \underline{z} \in \Omega)$ with $\boldsymbol{\sigma}(\underline{z})$ and $\varepsilon(\underline{z})$ as the local stress and strain fields, respectively. By using the superposition principle and taking the average of the local strain over $\Omega$, the general effective (homogenized) elasticity tensor is expressed as follows:

$$
\mathbb{C}^{\text {hom }}=\mathbb{C}^{s}+\sum_{r=1}^{N} \varphi^{r}\left(\mathbb{C}^{c, r}-\mathbb{C}^{s}\right): \mathbb{A}^{c, r}
$$

where $\varphi^{r}$ is the volume fraction representing the concentration of the inclusion family $r . \mathbb{A}^{c, r}$ is so-called strain concentration tensor which relates in a linear way the local strain $\varepsilon$ to the macroscopic uniform strain $\boldsymbol{E}$.

The determination of Eq. (1) is then summarized in determining the fourth order concentration tensor $\mathbb{A}^{c, r}$ for which we can benefit from the basic solution to the matrix-inclusion system of Eshelby's problem. For many engineering materials, it is useful to take into account both the influences of the shape of inclusions and those of its spatial distribution. For this purpose, we are interested in the homogenization scheme developed by Ponte-Castaneda and Willis in which two independent tensorial functions $\mathbb{P}_{\epsilon}^{r}$ (so-called Hill's tensor) and $\mathbb{P}_{d}$ are introduced to take into account the influences of the shape form of inclusions and of its spatial distribution on the material effective properties [1]. The corresponding effective (homogenized) stiffness tensor reads:

$$
\mathbb{C}^{\text {hom }}=\mathbb{C}^{s}-\mathbb{C}^{d}:\left(\mathbb{I}+\mathbb{P}_{d}: \mathbb{C}^{d}\right)^{-1}
$$


where we have in the Eshelby tensor-based form $\left(\mathbb{S}_{\epsilon}=\mathbb{P}_{\epsilon}: \mathbb{C}^{s}\right)$ :

$$
\mathbb{C}^{d}=\sum_{j=1}^{N} \varphi^{j}\left(\mathbb{C}^{s}-\mathbb{C}^{c, j}\right):\left[\mathbb{I}-\mathbb{S}_{\epsilon}^{j}:\left(\mathbb{I}-\mathbb{S}^{s}: \mathbb{C}^{c, j}\right)\right]^{-1} .
$$

For simplicity, we adopt in this study a spherical distribution for all cracks families, which returns in considering an isotropic form for $\mathbb{P}_{d}$ as follows:

$$
\mathbb{P}_{d}=\frac{\alpha}{3 k^{s}} \mathbb{J}+\frac{\beta}{2 \mu^{s}} \mathbb{K} ; \quad \text { with } \quad \alpha=\frac{3 k^{s}}{3 k^{s}+4 \mu^{s}} ; \quad \beta=\frac{6\left(k^{s}+2 \mu^{s}\right)}{5\left(3 k^{s}+4 \mu^{s}\right)} .
$$

\subsection{Application to Cracked Mediums}

We are now interested in microcracked materials. A family of cracks supposed to be penny-shaped in this work can be approximated as flat ellipsoids characterized by its unit normal vector (orientation) $\underline{n}$ and the aspect ratio $\frac{c}{a}$, noted $\epsilon$, with $a$ as the radius of the circular crack and $c$ as the half-length of the small axis. In addition, it is possible do express the volume fraction $\varphi^{r}$ of the $r^{t h}$ cracks family in the form:

$$
\varphi^{r}=\frac{4}{3} \pi a_{r}^{2} c_{r} \mathcal{N}_{r}=\frac{4}{3} \pi \epsilon d^{r}
$$

where $\mathcal{N}_{r}$ denotes the cracks density (number of cracks per unit volume) of the family $r$, and $d^{r}=\mathcal{N}_{r} a_{r}^{3}$ is the crack damage parameter widely used as internal variables in micromechanical analysis [12].

The elasticity tensor $\mathbb{C}^{c}$ for open cracks is classically defined by $\mathbb{C}^{c}=0$ to account for the cancellation of the stress on the crack faces. On the basis of the definition for damage parameters, the forth order tensor $\mathbb{C}^{d}$ in (3) is rewritten in the following form:

$$
\mathbb{C}^{d}=\sum_{j=1}^{N} d^{r} \mathbb{T}^{r}
$$

where, considering the assumption of small aspect ratio $(\epsilon \ll 1), \mathbb{T}^{r}$ reads for open cracks

$$
\mathbb{T}^{r}=\frac{4}{3} \pi \mathbb{C}^{s}: \lim _{\epsilon \rightarrow 0} \epsilon\left(\mathbb{I}-\mathbb{S}_{\epsilon}^{r}\right)^{-1}
$$

The expression of $\mathbb{T}^{r}$ in the Walpose's base are given in 13. For simplifying later formulations, it is convenient to rewrite Eq.(2) in the form:

$$
\mathbb{C}^{\text {hom }}=\mathbb{C}^{s}-\mathbb{C}^{d}+\mathbb{C}^{d}: \mathbb{B}: \mathbb{C}^{d}
$$

in which we put $\mathbb{B}=\left(\mathbb{I}+\mathbb{P}_{d}: \mathbb{C}^{d}\right)^{-1}: \mathbb{P}_{d}$. The macrosocpic stress-strain relation is then given by derivation of the macroscopic free energy $W\left(=\frac{1}{2} \boldsymbol{E}: \mathbb{C}^{\text {hom }}: \boldsymbol{E}\right)$ with respect to $\boldsymbol{E}$ :

$$
\boldsymbol{\Sigma}=\frac{\partial W}{\partial \boldsymbol{E}}=\mathbb{C}^{\text {hom }}: \boldsymbol{E}
$$


The conjugate thermodynamic force associated with any damage variable $d^{r}$ is defined by:

$$
\begin{aligned}
F^{d^{r}}=\frac{1}{2} \boldsymbol{E}: \mathbb{T}^{r}: \boldsymbol{E}-\frac{1}{2} \boldsymbol{E}:\left(\mathbb{T}^{r}: \mathbb{B}: \mathbb{C}^{d}\right. & \left.+\mathbb{C}^{d}: \mathbb{B}: \mathbb{T}^{r}\right): \boldsymbol{E} \\
& +\frac{1}{2} \boldsymbol{E}: \mathbb{C}^{d}: \mathbb{B}: \mathbb{T}^{r}: \mathbb{B}: \mathbb{C}^{d}: \boldsymbol{E} .
\end{aligned}
$$

\subsection{Damage Criterion and Evolution Law}

In the framework of thermodynamics, the damage criterion should be determined as a function of the conjugate force $F^{d^{r}}$ on the basis of experimental evidences. However the determination of such a criterion is usually not easy. Note that the expression of $F^{d^{r}}$ contains two linear terms in $\mathbb{T}^{r}, \mathbb{C}^{d}$ and $\mathbb{B}$, and one high order term. For the sake of simplification, only the two linear terms are used in damage driving force.

Denoting $\tilde{F}^{d^{r}}$ the first two terms of (10)

$$
\tilde{F}^{d^{r}}=\frac{1}{2} \boldsymbol{E}: \mathbb{T}^{r}: \boldsymbol{E}-\frac{1}{2} \boldsymbol{E}:\left(\mathbb{T}^{r}: \mathbb{B}: \mathbb{C}^{d}+\mathbb{C}^{d}: \mathbb{B}: \mathbb{T}^{r}\right): \boldsymbol{E},
$$

the following simple damage criterion is proposed:

$$
f\left(\tilde{F}^{d^{r}}, \underline{d}\right)=\tilde{F}^{d^{r}}-\mathcal{R}\left(d^{r}\right) \leq 0
$$

where $\mathcal{R}\left(d^{r}\right)$ is the local resistance against the damage propagation. $\mathcal{R}\left(d^{i}\right)=$ $c_{0}+c_{1} d^{r}$ is adopted in this work, with $c_{0}$ and $c_{1}$ being two material constants.

Following the theoretical framework of generalized standard materials, we adopt the normality rule in determination of damage evolutions:

$$
\dot{d}^{r}=\dot{\lambda}^{d^{r}} \frac{\partial f^{r}\left(\tilde{F}^{d^{r}}, d^{r}\right)}{\partial \tilde{F}^{d^{i}}}=\dot{\lambda}^{d^{r}} \quad ; \quad \dot{\lambda}^{d^{r}} \geq 0
$$

where the damage multiplier $\dot{\lambda}^{r}$ is determined by the consistency conditions $\dot{f}^{r}=0, r=1, \ldots, N$ for all considered families [13].

\section{Nonlocal Formulation}

It is commonly recognized that the strain softening or damage-induced strength degradation can be localized into a band of zero thickness with paradoxical consequences of structural failure with zero energy dissipation. In order to overcome this shortcoming in continuum models without characteristic length, the so-called non local approaches are widely used. The basic idea consists in replacing the local damage force $\tilde{F}^{d^{r}}$ for all considered families with its average over a representative volume $V$ of the material centered at a given point [14]. The damage variable $d^{r}$ is then function of the non local driving force $\overline{\tilde{F}^{d^{r}}}$ which will be defined as:

$$
\overline{\tilde{F}^{d^{r}}}(x)=\int_{V} \varpi(x, y) \tilde{F}^{d^{r}}(y) d V(y) .
$$


In Eq.(14), $\varpi(x, y)$ is a space weight function which describes the mutual nonlocal interactions and depends only on the distance between the source point $x$ and the receiver point $y$. Mathematically, the normalization condition $\int_{V} \varpi(x, y) d V=$ 1 is required for a uniform field. In this study, we adopt the following Gauss-type weighting function,

$$
\varpi(x, y)=\left(\frac{1}{l \sqrt{2 \pi}}\right)^{N_{d i m}} \exp \left(-\frac{\|x-y\|^{2}}{2 l^{2}}\right)
$$

where $N_{\text {dim }}$ is the number of spatial dimensions and $l$ is a material characteristic length which defines the size of interaction zone for failure processes. The factor $\left(\frac{1}{l \sqrt{2 \pi}}\right)^{N_{d i m}}$ is used to satisfy the normalization condition.

\section{Numerical Applications}

\subsection{3-Point Bending Test}

Three-point bending test represents a structural case which may emphasize more practical issues in terms of complexity and size when it comes to industrial structures. The geometry and the loading condition of this test are shown in Fig. 1. The parameters used in the analysis are as follows: $E^{s}=3.7 \times 10^{4} \mathrm{MPa}$, $c_{0}=1 . \times 10^{-3} \mathrm{~J} . \mathrm{m}^{-2}$ and $c_{1}=5 . \times 10^{-4} \mathrm{~J} . \mathrm{m}^{-2}$. The structure is discretesized respectively by 560 and 990 rectangle elements with 320 and 504 elements in the zone $|x| \leq 50 \mathrm{~mm}$, respectively. The force-displacement relations and the global damage distribution diagrams for the two meshes are shown and compared respectively in Fig. 22 and Fig. 3, from which mesh independent properties are observed. This kind of result is in agreement with most experimental data reported in literature.

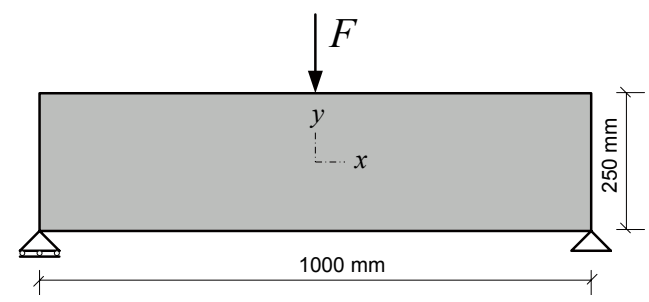

Fig. 1. Geometry and loading condition of the 3-point bending test

\subsection{Hassanzadeh's Direct Tension Test}

The second numerical application of the proposed model concerns a direct traction test performed by Hassanzadeh on a four-side notched concrete sample [15. The geometric description of the notched sample and the loading condition are 


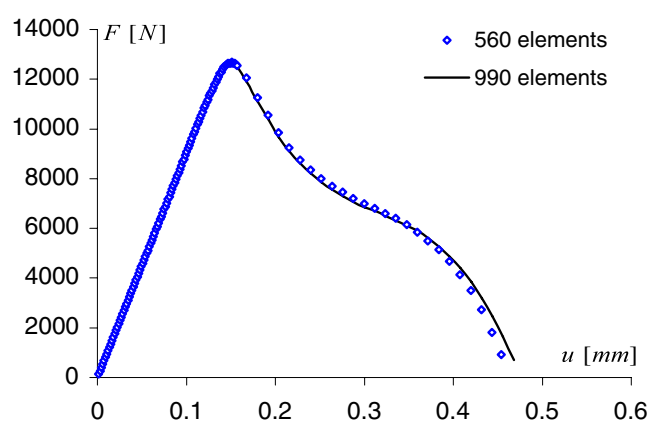

Fig. 2. Comparisons of the force-displacement response curves

a)

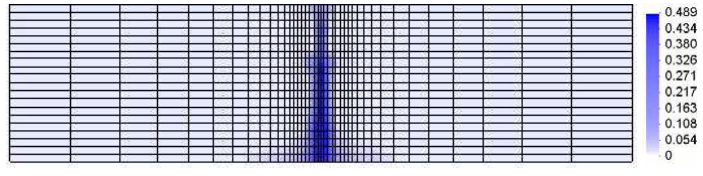

b)

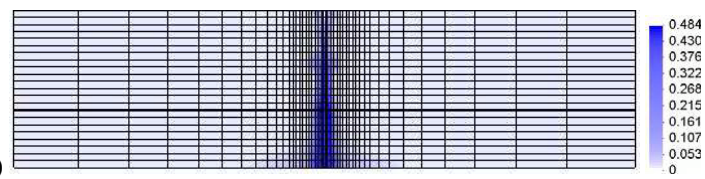

Fig. 3. Final global damage distributions for the two meshes: a) 560 meshes and b) 990 meshes
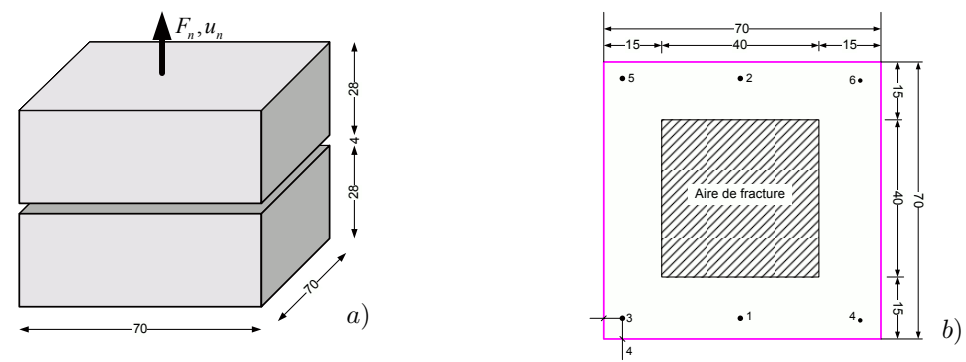

Fig. 4. Geometry (in mm) and loading condition of the Hassanzadeh test: a) geometry of the structure; b) geometry of the notches

indicated in Fig. 4. The hypothesis of plane strain condition is adopted for the numerical analysis.

In order to study the mesh sensitivity of the proposed nonlocal micromechanics based model, two different meshes are considered: the first discretization with 960 rectangular elements and the second one with 1512 elements which is obtained by refining the discretization in the central fracture zone. The material 


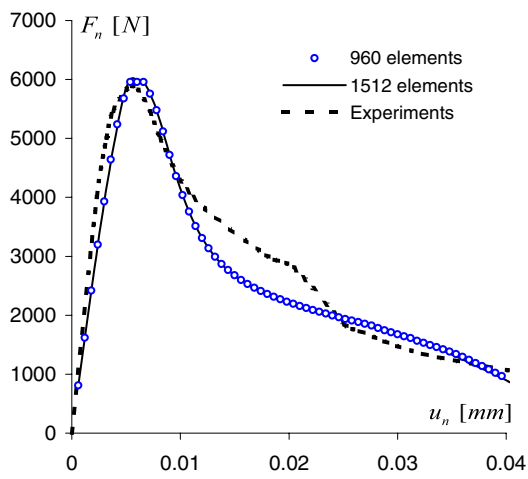

Fig. 5. Force-displacement response curves and comparisons to the experimental data reported in 15 .

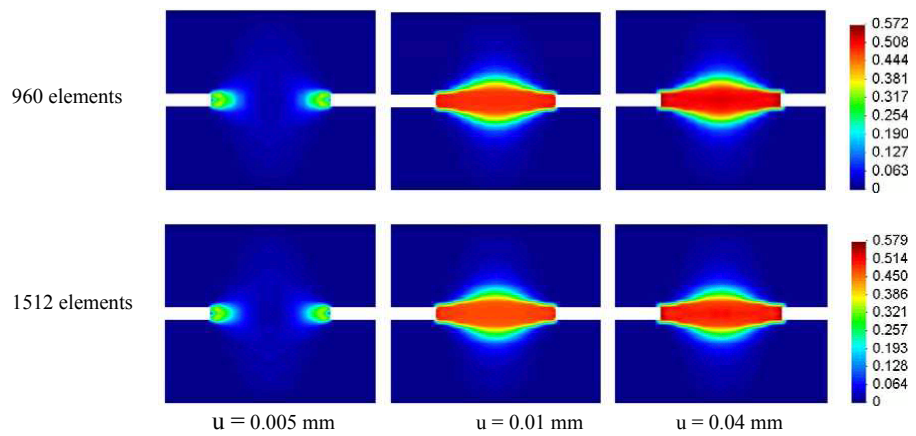

Fig. 6. Distributions of the global damage at three displacement levels for the two meshes

constants and model parameters are: $E^{s}=3.6 \times 10^{4} \mathrm{MPa}, c_{0}=7.5 \times 10^{-4} \mathrm{~J}^{\mathrm{m}} \mathrm{m}^{-2}$ and $c_{1}=1 . \times 10^{-3}{\mathrm{~J} . \mathrm{m}^{-2}}$.

Fig. 5 shows the force-displacement curves for the two meshes which are compared to the experimental data reported by Hassanzadeh [15. The global damage distributions at three different values of imposed displacements are presented and compared for the considered meshes in Fig. 6. It can be seen a good agreement between the numerical results and experimental data. Further, the mesh independency of numerical prediction is again observed.

\section{Conclusions}

In this study, we present a new homogenization-based damage model with few parameters. The micromechanical grounds provide the proposed model the capacity of dealing with main physical aspects such as unilateral effects related to cracks closures, the crack spatial distribution and interaction effects between mi- 
crocracks, which are generally neglected in widely-used macroscopic models. The constitutive model is extended to the nonlocal version and implemented into a finite element program. The numerical applications to concrete structures show that the proposed micromechanics based model provides a promising tool to further capture and model the complex mechanical behaviors and failure process in quasi brittle materials.

\section{References}

1. Kranz, R.L.: . Microcracks in rocks: a review, Tectonophysics, 100(1983) 449-480

2. Zhao, Y.: Crack pattern evolution and a fractal damage constitutive model for rock. International Journal of Rock Mechanics and Mining Sciences, 35(1998) 349-366 W

3. Murakami, S., Kamiya,K.: . Constitutive and damage evolution equations of elastic brittle materials based on irreversible thermodynamics. International Journal of Mechanical Science. 39(1996) 473-486

4. Halm, D., Dragon, A.: A model of anisotropic damage by mesocrack growth: unilateral effect. Int. J. Damage Mech. 5(1996) 384-402

5. Swoboda, G., Yang, Q.: An energy-based damage model of geomaterials I and II: deduction of damage evolution laws. Int. J. Solids Structures. 36(1999) 1735-1755

6. Dragon, A., Halm, D.: Damage Mechanics. Some modelling challenges. Institute of Fundamental Technological Research, Polysh Academy of Science, Editeur: IPPT PAN, Warsaw, Poland. (2004)

7. Andrieux, S., Bamberger, Y., Marigo, J.J.: Un modèle de matériau microfissuré pour les roches et les bétons. Journal de mécanique théorique et appliquée. 5(1986) 471-513

8. Gambarotta, L., Lagomarsino, S.: A microcrak damage model for brittle materials. International Journal of Solids and Structures. 30(1993) 177-198

9. Pensée, V., Kondo, D., Dormieux, L.: Micromechanical analysis of anisotropic damage in brittle materials. Journal of Engineering Mechanics, ASCE. 128(2002) 889897

10. Eshelby, J.D.: The determination of the elastic field of an ellipsoidal inclusion and related problems. Proc. R. Soc. Lond. A. 241(1957) 375-396

11. Ponte-Castaneda, P., Willis, J.R.: The effect of spatial distribution on the behavior of composite materials and cracked media. Journal of the Mechanics and Physics of Solids. 43(1995) 1919-1951

12. Budiansky, B., O'Connel, J.R.: Elastic moduli of a cracked solid. International Journal of Solids and Structures. 12(1976) 81-97

13. Zhu, Q.Z.: Applications des approches d'homogénéisation à la modélisation tridimensionnelle de l'endommagement des matériaux quasi fragile : Formulation, validation et implémentation numérique. Ph.D. Thesis (in French), University of Lille I, France. (2006)

14. Pijaudier-Cabot, G., Bazant, Z.P.: Nonlocal Damage Theory. Journal of Engineering Mechanics. 113(1987) 1512-1533

15. Hassanzadeh, M.: Behavior of fracture process zones in concrete influenced by simultaneously applied normal and shear displacements, Ph.D. Thesis Lund Institute of Technology, Lund. (1991) 